\title{
Editorial \\ Introducing Breast Cancer Research's updates on clinical trials
}

Martine Piccart ${ }^{1}$ and Eric Winer ${ }^{2}$

\author{
1Jules Bordet Institute, Brussels, Belgium \\ 2Dana-Farber Cancer Institute, Boston, Massachusetts, USA \\ Corresponding author: Eric Winer, Eric_Winer@dfci.harvard.edu \\ Published: 10 June 2004 \\ Breast Cancer Res 2004, 6:164 (DOI 10.1186/bcr810) \\ (C) 2004 BioMed Central Ltd
}

In the present issue of Breast Cancer Research, we are launching a new feature focused on clinical research in breast cancer. Each issue will contain an update from one of the major worldwide groups that focuses on their most important clinical trials. The objectives and scientific rationale for the trial(s) will be described, and the status of the trial(s) (present accrual, planned closure date) will also be provided. We are pleased that the European Organisation for Research and Treatment of Cancer (Belgium) was willing to be the first in this series.

We have two goals in mind in promoting this new feature. First, we want to provide a forum for each of the cooperative groups to share their current research. In this way, clinicians and researchers around the world can become familiar with the questions that are being addressed in ongoing trials. These updates may, in some cases, lead to collaborations across groups. The recent and ongoing experience with the trials in premenopausal women with hormone receptor-positive tumors has demonstrated the feasibility of conducting clinical trials (SOFT, A Phase III Trial Evaluating the Role of Ovarian Function Suppression and the Role of Exemestane as Adjuvant Therapies for Premenopausal Women with Endocrine Responsive Breast Cancer; TEXT, A Phase III Trial Evaluating the Role of Exemestane Plus GnRH Analogue as Adjuvant Therapy for Premenopausal Women with Endocrine Responsive Breast Cancer; and PERCHE, A Phase III Trial Evaluating the Role of Chemotherapy as Adjuvant Therapy for Premenopausal Women with Endocrine Responsive Breast Cancer Who Receive Endocrine Therapy ) on both sides of the Atlantic Ocean. Furthermore, we hope these clinical trial updates may lead to enhanced patient accrual through awareness and referrals.

The second goal is even more important. Despite improvements in breast cancer screening, local therapy, and adjuvant systemic therapy, far too many women ultimately die from breast cancer. Over the past decade, there has been an unprecedented expansion in our understanding of the basic mechanisms underlying breast cancer. There is greater knowledge than ever before about growth factor receptors, signaling pathways, tumorstromal interactions, and a whole range of other topics that could lead to therapeutic advances. It is our view that a substantial reduction in breast cancer mortality is critically dependent on close collaborations between basic, translational, and clinical researchers. By publishing updates of the ongoing trials, we hope to bring basic and translational scientists who work on breast cancer closer to the clinic by exposing them to the unanswered clinical questions. At the same time, we challenge clinicians and clinical trialists to interact with their colleagues in the laboratory and to design trials that will help answer questions that arise from laboratory-based hypotheses.

At some point in the not so distant future, mortality from breast cancer will be a concept of the past. But approaching this goal in the next decade will depend upon close and thoughtful collaborations between those of us who see patients and design trials, and our colleagues who are working in the laboratory.

We hope the series will be of interest to the readership of Breast Cancer Research. Over the months ahead, we welcome and look forward to your comments on how we can improve this effort.

\section{Competing interests}

None declared. 